\title{
Leveraging circuit theory and nonlinear dynamics for the efficiency improvement of energy harvesting
}

\author{
Michele Bonnin $₫$ Fabio L. Traversa $₫$ - Fabrizio Bonani $₫$
}

Received: 17 September 2020 / Accepted: 6 February 2021 / Published online: 25 February 2021

(C) The Author(s) 2021

\begin{abstract}
We study the performance of vibrational energy harvesting systems with piezoelectric and magnetic inductive transducers, assuming the power of external disturbance concentrated around a specific frequency. Both linear and nonlinear harvester models are considered. We use circuit theory and equivalent circuits to show that a large improvement in both the harvested energy and the power efficiency is obtained, for linear systems, through a proper reactive modification of the load. For nonlinear systems, we use methods of nonlinear dynamics to derive analytical formulae for the output voltage, the harvested energy and the power efficiency. We show that also for the nonlinear case, the modified load significantly boosts the performance.
\end{abstract}

Keywords Energy harvesting · Nonlinear oscillators · Equivalent circuits · Load matching · Power efficiency · Nonlinear resonance

\footnotetext{
M. Bonnin $(\varangle) \cdot$ F. Bonani

Dipartimento di Elettronica e Telecomunicazioni,

Politecnico di Torino, Turin, Italy

e-mail: michele.bonnin@polito.it

F. Bonani

e-mail: fabrizio.bonani@polito.it

F. L. Traversa

MemComputing Inc., San Diego, CA, USA

e-mail: ftraversa@memcpu.com
}

\section{Introduction}

Bolstered by the rapid deployment of the Industry 4.0 paradigm, the proliferation of Internet of Things (IoT) applications is expected to reach 31 billion connected devices by 2020, and 75 billion devices by 2025 [1]. At the very foundation of IoT lies the idea that a network of devices with embedded electronics, sensors, actuators and software are connected and interact via the Internet [2-4]. Among the many challenges posed by IoT, the problem of how to supply power to this network of wireless nodes is of paramount importance. Although the power needed to operate each device is small, typically in the microwatt to milliwatt range, their huge number, their often remote and difficult to access location and their ongoing miniaturization make old fashioned solutions, e.g., batteries, quite rarely a viable approach. A striking example are wearable systems, which are both portable and wireless, and thus where batteries would be an obvious choice. Unfortunately, the progress in energy storage density has, for the moment, been unable to keep the pace and batteries remain, with respect to other electronic components, bulky and heavy $[5,6]$.

A significant literature body, see, e.g., [7-9], suggests that electronic systems capable to wirelessly exchange not only information but also energy may represent a valid alternative. Yet another solution is to design systems capable of self-powering, by scavenging energy from the surrounding environment. The collected power can be used as a direct power source, 
or at least to extend the battery lifespan for those systems where continuous operation without maintenance is required. The set of technical solutions exploiting this idea is referred to as energy harvesting [10-12].

Energy harvesting (EH) technologies exploit different physical principles, depending upon the external power source to be tapped. Irrespective of the working principle, a common issue of $\mathrm{EH}$ apparatuses is the limited power density of the source. However, although negligible at the macroscale, energy dispersed in the environment is significant at the microscale, making $\mathrm{EH}$ technologies a viable solution to powering small autonomous devices.

Among the many possible sources, kinetic energy plays a very prominent role, because of its comparatively large power density and its widespread availability. Kinetic energy exists in the form of mechanical vibrations, regular or random displacements and driving forces. It can be found in mechanical structures, due to impacts or periodic motions, in buildings and bridges, due to traffic and wind, in vehicles, due to road asperity and engine induced vibrations, as well as in human body motion [13-16].

Kinetic energy harvesters rely on some kind of oscillating structure to capture vibrations, and on a transducer that converts kinetic energy into electrical power. Linear energy harvesters require a fine tuning of the oscillator's resonant frequency, to match the spectral range of environmental vibrations where most of the energy is concentrated. In many applications, this is a key limiting factor, due to geometrical and dimensional constraints. In fact, the rule of thumb is that the smaller is the size of an object, the larger its resonant frequency will be. Thus, it is very difficult to design energy harvesters that are both miniaturized and that work efficiently at low frequencies, making, for instance, the realization of wearable electronics that scavenge energy from human body motion very challenging. In this respect, it has been suggested that nonlinear oscillators can perform better than linear ones [17-22]. When compared to their linear counterparts, nonlinear energy harvesters show a wider steady-state frequency bandwidth and may exhibit multi-stability and even chaotic dynamics, thus suggesting that they can be more efficient especially in random and nonstationary vibratory environments [23-26].

Oscillators periodically exchange energy between two different forms. The losses taking place during each oscillation cycle, both dissipated by internal resistances and delivered to the load, can be significantly less than the total energy involved in the oscillation. For example, an $L C$ oscillator characterized by a quality factor $Q$ loses approximately a fraction $1 / Q$ of its energy per oscillation cycle. The remaining power bounces back and forth between the source and the load. It is well known from circuit theory that, in order to maximize the power efficiency, it is necessary to minimize the power that merely flows in the circuit with no useful contribution, a procedure known as power factor correction.

In this paper, we apply circuit theory and nonlinear dynamics methods to improve the efficiency of $\mathrm{EH}$ systems. We study the equivalent circuits (ECs) for two well-known energy harvesters: piezoelectric and magnetic inductive. Using circuit theory, we show that for linear harvesters, a simple modification of the load inspired by previous works [27-31] allows to improve the maximum collected power and the power efficiency. In the nonlinear case, using nonlinear dynamics methods we solve the state equations and find analytical formulae for the harvested power and the power efficiency. We show that, similarly to the linear case, properly modifying the load increases both the harvested power and the power efficiency.

The paper is organized as follows. In Sect. 2, we present the harvester models and ECs for both piezoelectric- and magnetic induction-based devices, showing how nonlinearities in the mechanical part (stiffness of the rod and in the springs, respectively) can be accounted for in the circuit equations. In Sect. 3, we investigate the linear limit. We apply standard circuit theory to solve the governing equations, and we show how the harvested energy and power efficiency can be maximized by a proper adaptation of the load. In Sect. 4, we study the nonlinear case. We show that the governing equations can be put in a standard form, valid for both the piezoelectric- and the magnetic inductancebased harvesters. We use tools of nonlinear dynamics to solve the state equations, and we derive analytical formulae for the harvested power and power efficiency. We show that even for the nonlinear harvester, the adapted load outperforms a purely resistive load. Finally, Sect. 5 is devoted to conclusions.

\section{Harvester modeling}

An EH device for mechanical vibration scavenging is composed by an oscillator that captures kinetic energy 


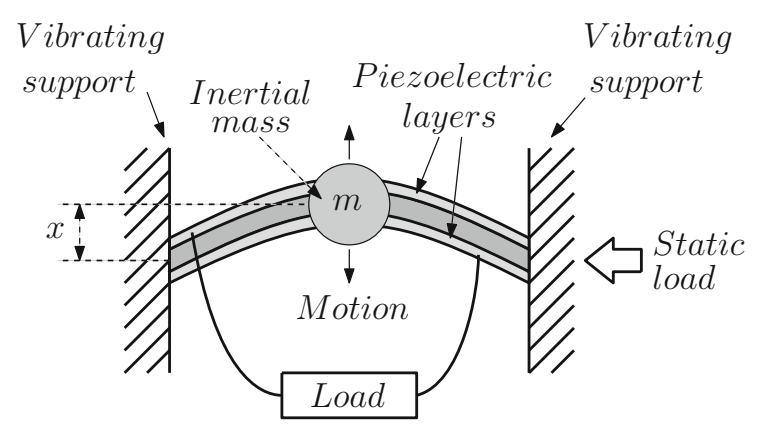

Fig. 1 Schematic representation of a piezoelectric energy harvester

from environmental vibrations, and by a transducer, responsible for the conversion of kinetic energy into usable electrical power. Transducers may exploit different physical principles for energy conversion including piezoelectric, magnetic induction and electrostatic transduction methods.

\subsection{Piezoelectric energy harvester}

A schematic representation of a piezoelectric energy harvester is shown in Fig. 1.

A simple piezoelectric energy harvester is composed by a cantilever beam, covered by a layer of piezoelectric material, fixed at one end to a moving support with an inertial mass $m$ at the opposite end to increase the oscillation amplitude. Vibrations of the support produce oscillations of the cantilever, inducing mechanical stress and strain in the piezoelectric material that are converted into electrical currents.

A more sophisticated setup is shown in Fig. 1 $[23,32]$. A thin beam covered by piezoelectric layers and with an inertial mass $m$ is clamped at both ends. An external static load can be applied to one end, to tune the natural frequency of the beam and to introduce a cubic nonlinearity. Under the influence of an external dynamic excitation, the beam undergoes oscillations around a static position defined by the axial load. If the mass of the cantilever and the effect of gravity are neglected, the equation of motion for the mechanical system reads $[18,33]$

$m \ddot{x}=-U^{\prime}(x)-\gamma \dot{x}-k_{v} v(t)+F_{\text {in }}$

where $x$ is the displacement from the vertical equilibrium position, $U(x)$ is the elastic potential of the can-

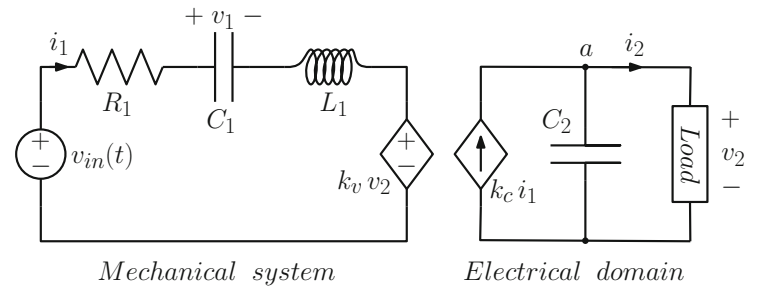

Fig. 2 EC for the piezoelectric harvester

tilever beam and the prime denotes the first derivative, $\gamma$ is the friction coefficient, $k_{v} v(t)$ describes the action of the piezoelectric layer onto the mechanical system $\left(v(t)\right.$ is the transducer voltage), and $F_{\text {in }}$ is the external perturbation.

The energy of ambient vibrations is typically spread over a wide frequency range, suggesting to model perturbation as white Gaussian noise. However, such random process can be represented as a superposition of harmonic (sinusoidal) signals with random phases and frequencies [34]. For the sake of simplicity, we consider here a vibration energy concentrated around a single frequency, and thus, we model vibrations as a simple harmonic driving force.

The piezoelectric device is characterized by an internal capacitance $C_{2}$, an internal resistance, here assumed to be negligible for the sake of simplicity, and a coupling with the mechanical part represented by parameter $k_{c}$.

The EC for the electromechanical system is shown in Fig. 2, where the parallel branches on the right represent the piezoelectric device, feeding a generic load.

Applying Kirchhoff voltage law (KVL) to the left loop yields

$\ddot{q}_{1}=-\frac{1}{L_{1}} v_{1}\left(q_{1}\right)-\frac{R_{1}}{L_{1}} \dot{q}_{1}-\frac{k_{v}}{L_{1}} v_{2}+\frac{1}{L_{1}} v_{\text {in }}$

where $\dot{q}_{1}=i_{1}(t)$, and $v_{1}\left(q_{1}\right)$ is the voltage-charge characteristic of the capacitor $C_{1}$. It is straightforward to verify that (1) and (2) are equivalent provided $x=q_{1}, m=L_{1}, U^{\prime}(x)=v_{1}\left(q_{1}\right), \gamma=R_{1}, F_{\text {in }}=v_{\text {in }}$ and $v=v_{2}$.

Application of Kirchhoff current law (KCL) to node $a$ on the right yields

$\dot{v}_{2}=\frac{k_{c}}{C_{2}} \dot{q}_{1}-\frac{1}{C_{2}} i_{2}$

Usually, purely resistive loads are considered [18, 33]. However, we will show that both the harvested power and the harvester efficiency can be significantly boosted if the load has a reactive component. 


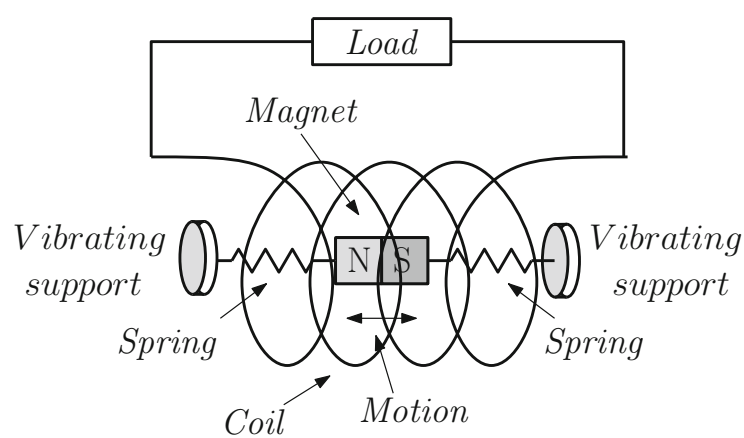

Fig. 3 Schematic representation of an electromagnetic energy harvester

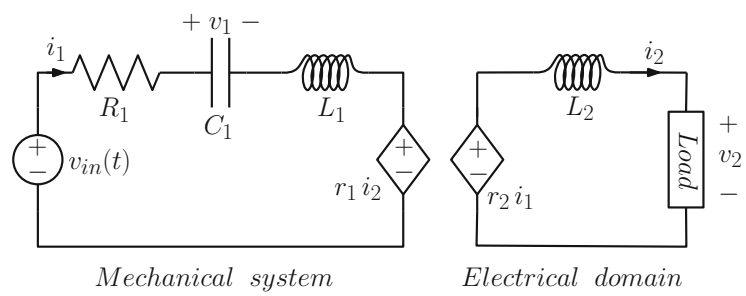

Fig. 4 EC for the electromagnetic harvester

\subsection{Electromagnetic energy harvester}

Examples of electromagnetic energy harvesters are given in $[35,36]$, with the schematic representation shown in Fig. 3. A small magnet with mass $m$ is placed inside a tube, connected to vibrating supports through springs, and with a coil wrapped around the tube. An alternative setup, where the magnet is suspended in the tube by a magnetic field generated by other magnets, is described in [19]. Vibrations of the supports produce oscillations of the suspended magnet, inducing a current in the surrounding coil through Faraday's law. The equation of motion for the magnet is

$m \ddot{y}=-U^{\prime}(y)-c \dot{y}-r_{1} i(t)+F_{\text {in }}$

where $y$ is the displacement, $U(y)$ is the elastic potential of the springs, $c$ is the friction constant, $r_{1} i(t)$ describes the interaction with the coil through magnetic induction, and $F_{\text {in }}$ is the external perturbation.

Figure 4 shows the EC for the electromagnetic harvester, where the loop on the right describes the magnetic induced current in the coil, providing power to the load.

Applying KVL to the loop on the left gives

$\ddot{q}_{1}=-\frac{1}{L_{1}} v_{1}\left(q_{1}\right)-\frac{R_{1}}{L_{1}} \dot{q}_{1}-\frac{r_{1}}{L_{1}} i_{2}+\frac{1}{L_{1}} v_{\text {in }}$ which maps onto (4) provided $y=q_{1}, m=L_{1}$, $U^{\prime}(y)=v_{1}\left(q_{1}\right), c=R_{1}, i=i_{2}$ and $F_{\text {in }}=v_{\text {in }}$

Applying KVL to the loop on the right yields

$\dot{i}_{2}=\frac{r_{2}}{L_{2}} \dot{q}_{1}-\frac{1}{L_{2}} v_{2}$

We notice that (2)-(3) and (5)-(6) are completely analogous.

\section{Small signal analysis}

We begin to study the small signal limit, where it can be assumed that all one-port components have linear characteristics.

\subsection{Piezoelectric energy harvester}

Consider the EC in Fig. 2. At steady state in the phasor domain, ${ }^{1}$ denoting by $Y_{2}$ the load admittance, the state equations read

$$
\left[\begin{array}{cc}
R_{1}+j X_{1} & k_{v} \\
-k_{c} & G_{2}+j B_{2}
\end{array}\right]\left[\begin{array}{l}
I_{1} \\
V_{2}
\end{array}\right]=\left[\begin{array}{c}
V_{\text {in }} \\
0
\end{array}\right]
$$

where $X_{1}$ is the reactance of the left loop

$X_{1}=\omega L_{1}-\frac{1}{\omega C_{1}}$

$\omega$ and $V_{\text {in }}$ are the angular frequency and amplitude of the external forcing term, respectively, while $G_{2}=$ $\operatorname{Re}\left\{Y_{2}\right\}$ is the conductance of the load, and $B_{2}=$ $\omega C_{2}+\operatorname{Im}\left\{Y_{2}\right\}$ is the susceptance of the right part.

The relevant transfer functions are

$$
\begin{aligned}
Y_{\text {in }}(\omega) & =\frac{I_{1}}{V_{\text {in }}}=\frac{G_{2}+j B_{2}}{\left(R_{1}+j X_{1}\right)\left(G_{2}+j B_{2}\right)+k_{v} k_{c}} \\
H(\omega) & =\frac{V_{2}}{V_{\text {in }}}=\frac{k_{c}}{\left(R_{1}+j X_{1}\right)\left(G_{2}+j B_{2}\right)+k_{v} k_{c}}
\end{aligned}
$$

Assuming that the resonator on the left works at the resonance frequency $\omega_{0}=1 / \sqrt{L_{1} C_{1}}$ and that $\omega_{0}=\omega$

\footnotetext{
${ }^{1}$ Derivation of the state equations in the mechanical-electrical domains for both the piezoelectric and the electromagnetic energy harvesters is given in "Appendix".
} 
to maximize energy collection, using phasors and (10), the average power delivered to the load is

$$
\begin{aligned}
P_{\text {out }} & =\frac{1}{2} \operatorname{Re}\left[V_{2} I_{2}^{*}\right]=\frac{1}{2} G_{2}\left|V_{2}\right|^{2}=\frac{1}{2} G_{2}|H|^{2}\left|V_{\text {in }}\right|^{2} \\
& =\frac{1}{2} \frac{G_{2} k_{c}^{2}}{K^{2}+R_{1}^{2} B_{2}^{2}}\left|V_{\text {in }}\right|^{2}
\end{aligned}
$$

where $K=R_{1} G_{2}+k_{v} k_{c}$. Conversely, using (9) the average power injected by the voltage source is

$$
\begin{aligned}
P_{\text {in }} & =\frac{1}{2} \operatorname{Re}\left[V_{\text {in }} I_{1}^{*}\right]=\frac{1}{2} \operatorname{Re}\left[Y_{\text {in }}^{*}\right]\left|V_{\text {in }}\right|^{2} \\
& =\frac{1}{2} \frac{G_{2} K+R_{1} B_{2}^{2}}{K^{2}+R_{1}^{2} B_{2}^{2}}\left|V_{\text {in }}\right|^{2}
\end{aligned}
$$

so that the power efficiency reads

$\eta_{\mathrm{pz}}=\frac{P_{\mathrm{out}}}{P_{\text {in }}}=\frac{G_{2} k_{c}^{2}}{G_{2} K+R_{1} B_{2}^{2}}$

As a result, both the average dissipated power $P_{\text {out }}$ and the power efficiency $\eta_{\mathrm{pz}}$ are maximized when the susceptance $B_{2}$ is null. ${ }^{2}$ This condition cannot be attained by a resistive load, as customarily assumed [20, 33,35-37]. Conversely, maximum dissipated power and efficiency are achieved if the load is composed by a resistor $R_{2}=1 / G_{2}$ connected in parallel with an inductor $L_{2}$ with inductance $L_{2}=1 /\left(\omega_{0}^{2} C_{2}\right)=$ $L_{1} C_{1} / C_{2}$, as shown in Fig. 8 b. In this case, the circuit in Fig. 2 is composed by two resonators with the same resonant frequency connected through the controlled sources.

A realistic transducer model must represent a passive element, so that it does not supply energy to the rest of the circuit. According to the passive sign convention [38], the average power dissipated by the controlled sources is

$$
\begin{aligned}
P_{k_{v}}+P_{k_{c}} & =\frac{1}{2}\left(k_{v}-k_{c}\right) \operatorname{Re}\left\{V_{2} I_{1}^{*}\right\} \\
& =\frac{1}{2}\left(k_{v}-k_{c}\right) \operatorname{Re}\left\{H(\omega) Y_{\text {in }}^{*}(\omega)\right\}\left|V_{\text {in }}\right|^{2}
\end{aligned}
$$

\footnotetext{
${ }^{2}$ We do not consider here the case $R_{1} \rightarrow 0 \Omega$. For $R_{1} \rightarrow 0$, $R_{2}$ would be the only resistive load in the circuit, and all active power supplied by the source would be delivered to the load, with $100 \%$ efficiency irrespective of the values of parameters $L_{1}, C_{1}$, $\mathrm{C}_{2}$ and $R_{2}$.
}

In particular, at the resonance frequency

$$
P_{k_{v}}+P_{k_{c}}=\frac{1}{2}\left(k_{v}-k_{c}\right) \frac{G_{2} k_{c}}{\left(R_{1} G_{2}+k_{v} k_{c}\right)^{2}}\left|V_{\text {in }}\right|^{2}
$$

Equation (15) implies that the transducer is passive if and only if $k_{v} \geq k_{c}$. Considering that dissipation is accounted for by $R_{1}$, it can be assumed that the transducer's energy balance is null for all frequencies, thus implying $k_{v}=k_{c}$. For this reason, in many models one parameter only is used to describe the transducer [23,31,39].

Maximum average dissipated power is obtained with the load conductance

$G_{2}=\frac{k_{v}^{2}}{R_{1}}$

corresponding to a maximum average absorbed power

$P_{\text {out }}^{\max }=\frac{\left|V_{\text {in }}\right|^{2}}{8 R_{1}}$

and to the expected 50\% efficiency [38].

Efficiency higher than $50 \%$ can obviously be attained, either decreasing $R_{1}$ ( $G_{2}$ being kept fixed) or vice versa increasing $R_{2}$ ( $R_{1}$ being kept fixed). In fact, from (13) with $B_{2}=0 \mathrm{~S}$ we have $\partial \eta_{\mathrm{pz}} / \partial R_{1}<0$ and $\partial \eta_{\mathrm{pz}} / \partial G_{2}<0$. In particular, in the limit $G_{2} \ll$ $k_{c} k_{v} / R_{1}$, we have $K \approx k_{v} k_{c}$ and $\eta_{\mathrm{pz}} \rightarrow 1$. However, higher efficiency can be attained only giving up the maximum power transfer to the load, as from (11) we find that $\partial P_{\text {out }} / \partial G_{2} \neq 0$ for $G_{2} \neq k_{c} k_{v} / R_{1}$.

Figure 5 shows the Bode diagram for the magnitude of transfer functions $Y_{\text {in }}(\omega)$ and $H(\omega)$ for the cases of a resistive and of a resistive-inductive load. Parameter values have been normalized (including time) so that $\omega_{0}=1 \mathrm{rad} / \mathrm{s}$.

Figure 6 shows the average dissipated (output) power $P_{\mathrm{out}}$ and the power efficiency $\eta_{\mathrm{pz}}$ as a function of the external forcing term frequency. For the resistive load case, the average dissipated power is maximized by a proper matching of the resonance frequency to the forcing term. This can be done, for instance, by an appropriate choice of the inertial mass $m$. However, power efficiency is maximum at zero frequency and decreases rapidly as the forcing frequency increases. On the contrary, the resistive-reactive load not only has higher average dissipated power but, more importantly, 

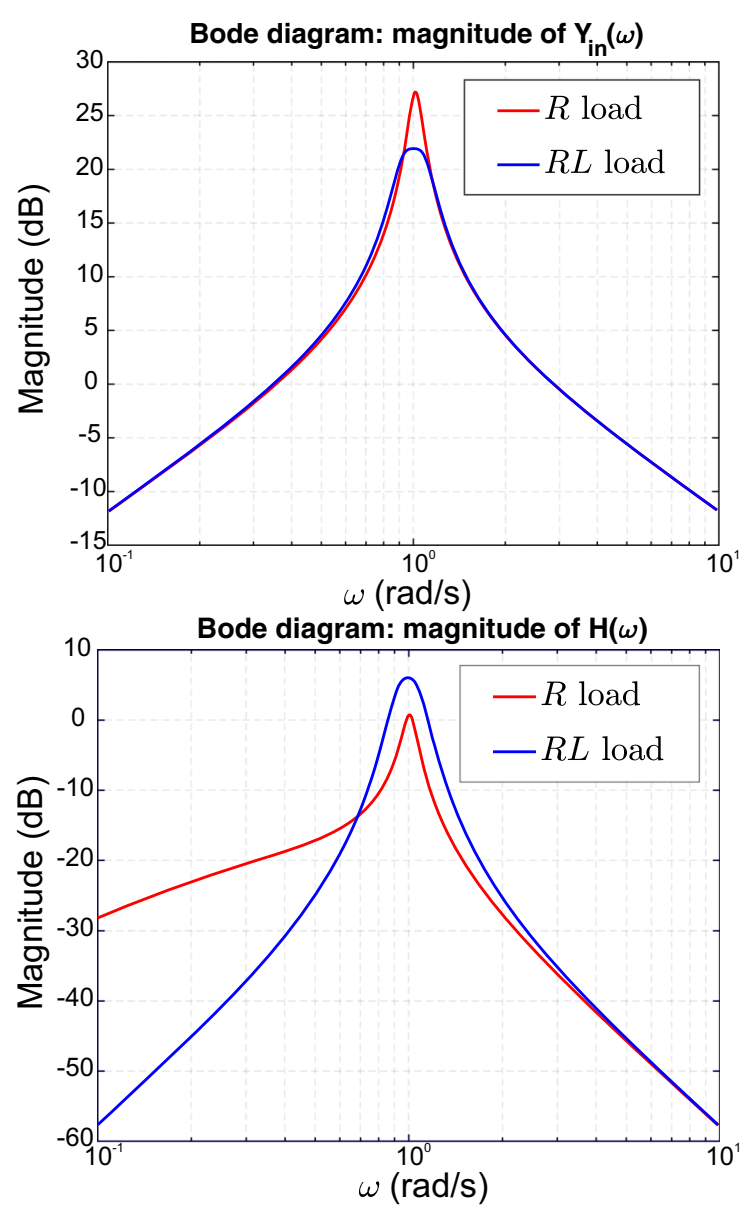

Fig. 5 Magnitude Bode diagram for transfer functions $Y_{\text {in }}(\omega)$ (above), and $H(\omega)$ (below), for a resistive (red) and a resistivereactive load (blue). Parameter values are $L_{1}=0.4 \mathrm{H} ; C_{1}=$ $2.5 \mathrm{~F} ; L_{2}=0.2 \mathrm{H} ; C_{2}=5 \mathrm{~F} ; R_{1}=0.04 \Omega ; G_{2}=1.5625 \mathrm{~S}$; $k_{v}=k_{c}=0.25$. (Color figure online)

both dissipated power and efficiency are maximum at the same frequency, provided that the mechanical and the electrical parts are tuned to the same resonance frequency. These findings are in agreement with previous works [31,39].

Finally, 7 shows the averaged power delivered to the load and the power efficiency, as functions of the inductance $L_{2}$ for the resistive-reactive load in Fig. 8 b. It can be seen that both $P_{\text {out }}$ and $\eta_{\mathrm{pz}}$ are maximum for $L_{2}=L_{1} C_{1} / C_{2}=0.2 \mathrm{~S}$, so that $B_{2}=0 \mathrm{~S}$. It can also be seen that both the output power and the efficiency converge to constant values for large $L_{2}$, corresponding to the values of a purely resistive load. (For $L_{2} \rightarrow+\infty$, the inductor is equivalent to an open circuit.)
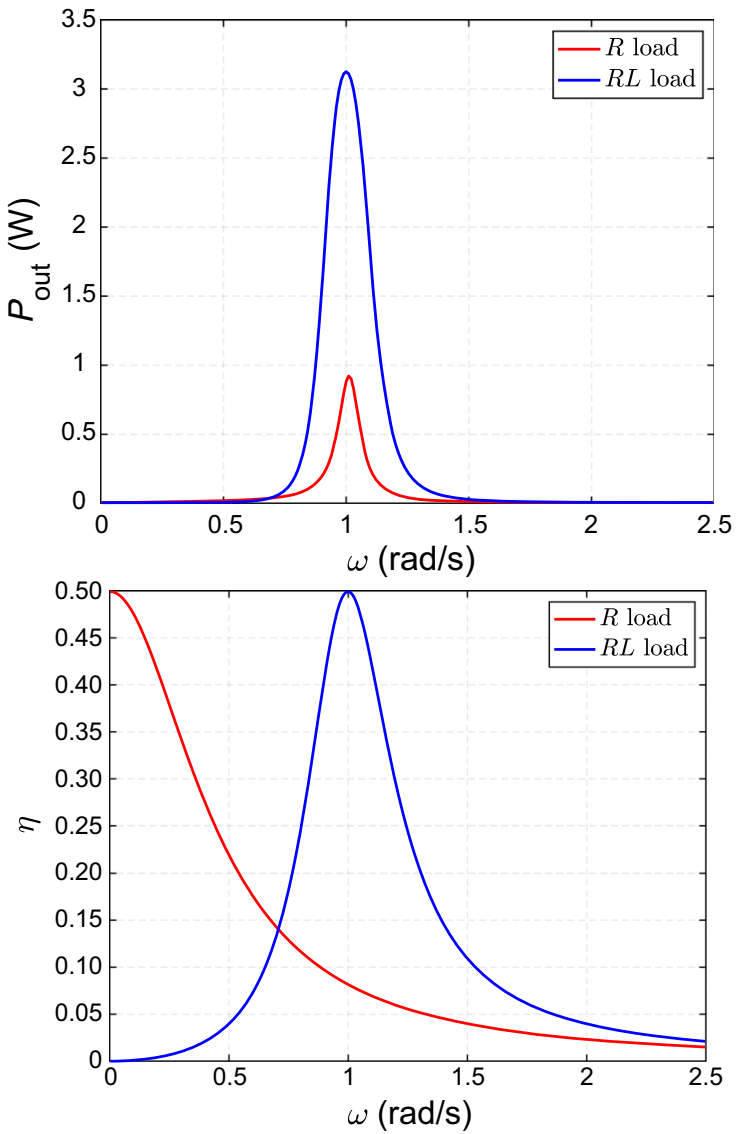

Fig. 6 Output average power (above) and power efficiency (below) for a resistive load (red) and a resistive-reactive load (blue) versus the frequency of the external forcing. Parameter values are the same used in Fig. 5. Output average power (above) is calculated assuming $V_{\text {in }}=1 \mathrm{~V}$. (Color figure online)

\subsection{Electromagnetic energy harvester}

The analysis of the electromagnetic energy harvester is completely analogous. Consider the EC shown in Fig. 4. Denoting as $Z_{2}$ the load impedance, at steady state in the phasor domain the state equations read

$\left[\begin{array}{cc}R_{1}+j X_{1} & r_{1} \\ -r_{2} & R_{2}+j X_{2}\end{array}\right]\left[\begin{array}{c}I_{1} \\ I_{2}\end{array}\right]=\left[\begin{array}{c}V_{\text {in }} \\ 0\end{array}\right]$

where $X_{1}$ and $X_{2}$ are the reactances of the left and right loops, respectively

$X_{1}=\omega L_{1}-\frac{1}{\omega C_{1}}$
$X_{2}=\omega L_{2}+\operatorname{Im}\left\{Z_{2}\right\}$ 

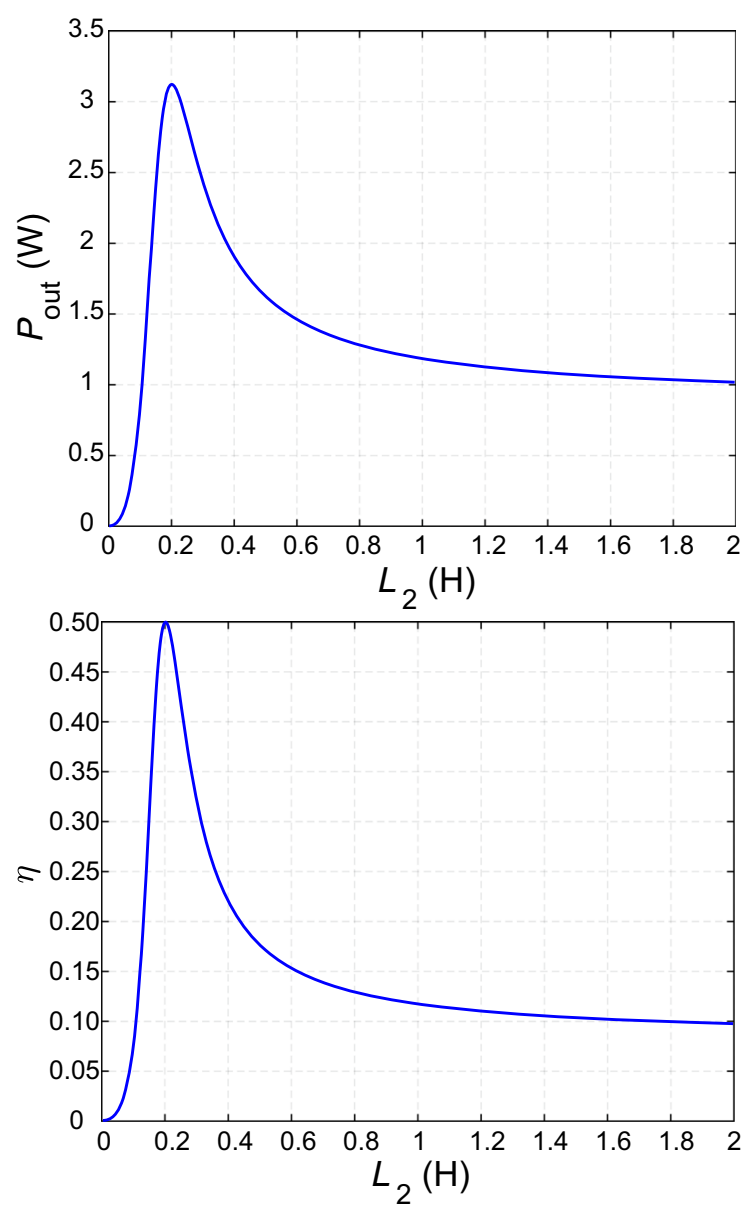

Fig. 7 Average (output) power delivered to the load (above), and power efficiency (below), as functions of the inductance $L_{2}$ for the resistive-reactive load in Fig. 8 b. Average output power (above) is calculated assuming $V_{\text {in }}=1 \mathrm{~V}$. Other parameter values are the same used in Fig. 5

and $R_{2}=\operatorname{Re}\left\{Z_{2}\right\}$.

The relevant transfer functions are

$$
\begin{gathered}
Y_{\text {in }}(\omega)=\frac{I_{1}}{V_{\text {in }}}=\frac{R_{2}+j X_{2}}{\left(R_{1}+j X_{1}\right)\left(R_{2}+j X_{2}\right)+r_{1} r_{2}} \\
Y_{\text {out }}(\omega)=\frac{I_{2}}{V_{\text {in }}}=\frac{r_{2}}{\left(R_{1}+j X_{1}\right)\left(R_{2}+j X_{2}\right)+r_{1} r_{2}}
\end{gathered}
$$

Assuming that the resonator on the left works at the resonance frequency and denoting by $R^{2}=R_{1} R_{2}+$ $r_{1} r_{2}$, the average power delivered to the load is
$P_{\text {out }}=\frac{1}{2} \frac{R_{2} r_{2}^{2}}{R^{4}+R_{1} X_{2}^{2}}\left|V_{\text {in }}\right|^{2}$,

whereas the average injected power is

$P_{\text {in }}=\frac{1}{2} \frac{R^{2} R_{2}+R_{1} X_{2}^{2}}{R^{4}+R_{1}^{2} X_{2}^{2}}\left|V_{\text {in }}\right|^{2}$

with power efficiency

$\eta_{\mathrm{em}}=\frac{R_{2} r_{2}^{2}}{R^{2} R_{2}+R_{1} X_{2}^{2}}$

Similarly to the piezoelectric energy harvester case, to maximize the average dissipated power and the power efficiency, the reactance $X_{2}$ must vanish. This can be obtained connecting a capacitor with capacitance $C_{2}=1 /\left(\omega_{0}^{2} L_{2}\right)=C_{1} L_{1} / L_{2}$, in series with the resistor, as shown in Fig. 8 c. In this way, the EC in Fig. 4 is composed of two resonators, connected through the controlled sources, characterized by the same resonance frequency $\omega_{0}$.

For the magnetic induction energy harvester, the average power dissipated by the transducer is

$$
\begin{aligned}
P_{r_{1}}+P_{r_{2}}= & \frac{1}{2}\left(r_{1} \operatorname{Re}\left\{I_{2} I_{1}^{*}\right\}-r_{2} \operatorname{Re}\left\{I_{1} I_{2}^{*}\right\}\right) \\
= & \frac{1}{2}\left(r_{1} \operatorname{Re}\left\{Y_{\text {out }}(\omega) Y_{\text {in }}^{*}(\omega)\right\}\right. \\
& \left.-r_{2} \operatorname{Re}\left\{Y_{\text {in }}(\omega) Y_{\text {out }}^{*}(\omega)\right\}\right)\left|V_{\text {in }}\right|^{2}
\end{aligned}
$$

At the resonance frequency

$$
P_{r_{1}}+P_{r_{2}}=\frac{1}{2}\left(r_{1}-r_{2}\right) \frac{R_{2} r_{2}}{\left(R_{1} R_{2}+r_{1} r_{2}\right)}\left|V_{\text {in }}\right|^{2}
$$

thus the transducer is passive for all frequencies if and only if $r_{1} \geq r_{2}$.

Assuming $r_{1}=r_{2}$, the maximum average dissipated power is obtained if the load has resistance

$R_{2}=\frac{r_{1}^{2}}{R_{1}}$

so that

$$
P_{\text {out }}^{\max }=\frac{\left|V_{\text {in }}\right|^{2}}{8 R_{1}}
$$




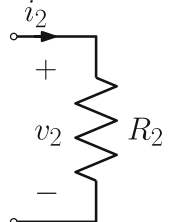

(a)

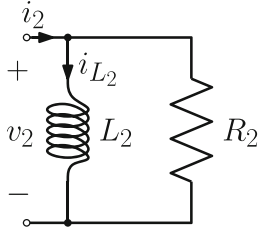

(b)

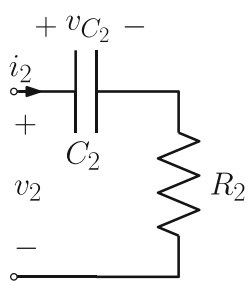

(c)
Fig. 8 Loads for the cases under consideration. a Resistive load considered in previous works. b Matched $R L$ load for the piezoelectric energy harvester. $\mathbf{c}$ Matched $R C$ load for the electromagnetic energy harvester

and the efficiency reaches the theoretical maximum of $50 \%$.

\section{Nonlinear energy harvester analysis}

The main drawback of linear energy harvesters is the limited working bandwidth. Linear resonators are designed to work efficiently at a specific, resonance frequency $\omega_{0}$. The amplitude response decreases quickly away from $\omega_{0}$, dropping dramatically outside the passband. Several works [17-22] suggest that nonlinear resonator-based harvesters may overcome this limitation. Exploiting the hysteretic amplitude response of a nonlinear resonator, it is in principle possible to increase the collected power, trading off the efficiency at a specific frequency against a larger bandwidth.

The idea is illustrated in Fig. 9, where the typical frequency response (amplitude versus frequency of the forcing term) of linear (black line) and nonlinear (blue and red lines) oscillators is compared. For similar values of the parameters, a linear oscillator in general exhibits an higher amplitude response than the nonlinear counterpart, but the amplitude decreases quickly out of a frequency interval, the passband width $B_{\mathrm{L}}$. Conversely, a nonlinear oscillator is in general characterized by a lower maximum amplitude, and by a wider passband width $B_{\mathrm{N}}$ where the amplitude response remains significant.

\subsection{Nonlinear piezoelectric energy harvester}

Consider the piezoelectric energy harvester, modeled by the EC shown in Fig. 2, with the resistive-inductive load in Fig. 8 b. Nonlinearity in the stiffness of the

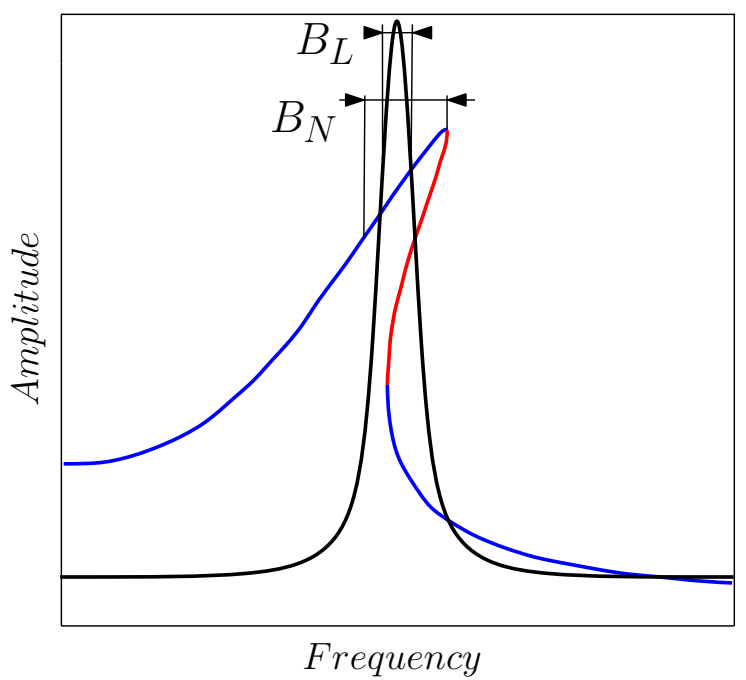

Fig. 9 Qualitative representation of the amplitude vs frequency response for a linear (black) and a nonlinear (blue-red) periodically driven resonator. Blue lines represent stable periodic responses, whereas the red line is an unstable response. $B_{\mathrm{L}}$ and $B_{\mathrm{N}}$ denote the passband width for the linear and the nonlinear resonator, respectively . (Color figure online)

beam is taken into account considering a capacitor with nonlinear voltage-charge characteristic. We assume a Duffing-type cubic stiffness term, describing the hardening spring effect observed in many mechanical systems [40]

$v_{1}\left(q_{1}\right)=\frac{1}{C_{1}} q_{1}+\frac{1}{\widetilde{C}_{1}} q_{1}^{3}$

Similarly, the inductor in the load is assumed to have a nonlinear current-flux characteristic, matching the nonlinearity of the capacitor

$i_{L_{2}}=\frac{1}{L_{2}} \phi_{2}+\frac{1}{\widetilde{L}_{2}} \phi_{2}^{3}$

Applying KVL to the left loop, KCL to node $a$, and rearranging the terms, we obtain the dynamic equations

$\ddot{q}_{1}+\frac{1}{L_{1} C_{1}} q_{1}=\frac{1}{L_{1}} v_{\text {in }}-\frac{R_{1}}{L_{1}} \dot{q}_{1}-\frac{1}{L_{1} \widetilde{C}_{1}} q_{1}^{3}-\frac{k_{v}}{L_{1}} \dot{\phi}_{2}$

$\ddot{\phi}_{2}+\frac{1}{L_{2} C_{2}} \phi_{2}=-\frac{G_{2}}{C_{2}} \dot{\phi}_{2}-\frac{1}{\widetilde{L}_{2} C_{2}} \phi_{2}^{3}+\frac{k_{c}}{C_{2}} \dot{q}_{1}$ 
Making the substitution $x_{1}=q_{1}, x_{2}=\phi_{2}$, assuming $v_{\text {in }}(t) / L_{1}=\varepsilon A \cos (\omega t)$ and introducing the new parameters ${ }^{3}$ :

$$
\begin{aligned}
\frac{1}{\sqrt{L_{1} C_{1}}} & =\omega_{1} & \frac{1}{\sqrt{L_{2} C_{2}}} & =\omega_{2} & & \frac{R_{1}}{L_{1}}=\varepsilon \gamma_{1} \\
\frac{G_{2}}{C_{2}} & =\varepsilon \gamma_{2} & \frac{1}{L_{1} \widetilde{C}_{1}} & =\varepsilon \delta_{1} & & \frac{1}{\widetilde{L}_{2} C_{2}}=\varepsilon \delta_{2} \\
\frac{k_{v}}{L_{1}} & =\varepsilon \sigma_{1} & \frac{k_{c}}{C_{2}} & =\varepsilon \sigma_{2} & &
\end{aligned}
$$

equations (32) and (33) become

$\ddot{x}_{1}+\omega_{1}^{2} x_{1}=\varepsilon\left(A \cos (\omega t)-\gamma_{1} \dot{x}_{1}-\delta_{1} x_{1}^{3}-\sigma_{1} \dot{x}_{2}\right)$

$\ddot{x}_{2}+\omega_{2}^{2} x_{2}=\varepsilon\left(-\gamma_{2} \dot{x}_{2}-\delta_{2} x_{2}^{3}+\sigma_{2} \dot{x}_{1}\right)$

\subsection{Nonlinear electromagnetic energy harvester}

Consider the electromagnetic energy harvester and its EC shown in Fig. 4, with the resistive-capacitive load in Fig. 8 c. In full analogy with the previous case, nonlinearity in the spring stiffness is accounted for by the nonlinear voltage-charge characteristic of the capacitor (30). We also assume that capacitor $C_{2}$ in the matched load has a similar characteristic

$v_{2}\left(q_{2}\right)=\frac{1}{C_{2}} q_{2}+\frac{1}{\widetilde{C}_{2}} q_{2}^{3}$

Applying KVL to both loops, and rearranging the terms, we obtain the equations

$\ddot{q}_{1}+\frac{1}{L_{1} C_{1}} q_{1}=\frac{1}{L_{1}} v_{i n}-\frac{R_{1}}{L_{1}} \dot{q}_{1}-\frac{1}{L_{1} \widetilde{C}_{1}} q_{1}^{3}-\frac{r_{1}}{L_{1}} \dot{q}_{2}$

$\ddot{q}_{2}+\frac{1}{L_{2} C_{2}} q_{2}=-\frac{R_{2}}{L_{2}} \dot{q}_{2}-\frac{1}{L_{2} \widetilde{C}_{2}} q_{2}^{3}+\frac{r_{2}}{L_{2}} \dot{q}_{1}$

Assuming again $v_{\text {in }}(t) / L_{1}=\varepsilon A \cos (\omega t)$, making the substitution $x_{i}=q_{i}$ and introducing the new parameters

$$
\frac{1}{\sqrt{L_{i} C_{i}}}=\omega_{i} \quad \frac{R_{i}}{L_{i}}=\varepsilon \gamma_{i} \quad \frac{1}{L_{i} \widetilde{C}_{i}}=\varepsilon \delta_{i} \quad \frac{r_{i}}{L_{i}}=\varepsilon \sigma_{i}
$$

\footnotetext{
${ }^{3}$ It is worth mentioning that introducing the parameter $\varepsilon$ is not strictly required. We use it to cast the following equations in the standard form adopted when averaging techniques are applied.
}

for $i=1,2$, we obtain, again, equations (34) and (35), although with a different meaning of variables and parameters.

Therefore, we can use the same dynamical system to study both the piezoelectric and the electromagnetic energy harvester.

\subsection{Analysis}

We shall find an approximate solution to equations (34)-(35) exploiting a perturbative method [40] adapted to the case under study. Let's rewrite the equations in the form

$\ddot{x}_{i}+\omega_{i}^{2} x_{i}=\varepsilon\left(A_{i} \cos (\omega t)-\gamma_{i} \dot{x}_{i}-\delta_{i} x_{i}^{3}-\sigma_{i} \dot{x}_{j}\right)$

with $i=1,2, j \neq i$ and

$A_{i}=\left\{\begin{array}{l}A \text { for } i=1 \\ 0 \text { for } i=2\end{array} \quad \sigma_{i}=\left\{\begin{array}{l}\sigma_{1} \text { for } i=1 \\ -\sigma_{2} \text { for } i=2\end{array}\right.\right.$

We introduce the invertible coordinate transformation ${ }^{4}$ $\left(\begin{array}{c}u_{i} \\ v_{i}\end{array}\right)=\left(\begin{array}{cc}\cos (\omega t) & -\frac{1}{\omega} \sin (\omega t) \\ -\sin (\omega t) & -\frac{1}{\omega} \cos (\omega t)\end{array}\right)\left(\begin{array}{c}x_{i} \\ \dot{x}_{i}\end{array}\right)$

In the new rotating reference frame, the unforced system is stationary. Taking time derivatives

$$
\begin{aligned}
& \dot{u}_{i}=-\frac{1}{\omega}\left(\ddot{x}_{i}+\omega^{2} x_{i}\right) \sin (\omega t) \\
& \dot{v}_{i}=-\frac{1}{\omega}\left(\ddot{x}_{i}+\omega^{2} x_{i}\right) \cos (\omega t)
\end{aligned}
$$

introducing $\Omega_{i}=\left(\omega_{i}^{2}-\omega^{2}\right) / \varepsilon$, and using (39) we obtain

$$
\begin{aligned}
\dot{u}_{i}= & -\frac{\varepsilon}{\omega}\left(A_{i} \cos (\omega t)-\gamma_{i} \dot{x}_{i}-\delta_{i} x_{i}^{3}-\Omega_{i} x_{i}-\sigma_{i} \dot{x}_{j}\right) \\
& \times \sin (\omega t) \\
\dot{v}_{i}= & -\frac{\varepsilon}{\omega}\left(A_{i} \cos (\omega t)-\gamma_{i} \dot{x}_{i}-\delta_{i} x_{i}^{3}-\Omega_{i} x_{i}+\sigma_{i} \dot{x}_{j}\right) \\
& \times \cos (\omega t)
\end{aligned}
$$

$\overline{{ }^{4} \text { Variables } v_{i}}$ used here should not be confused with voltages in the ECs. 
Inverting (41) and substituting into (44)-(45), we obtain a system of non-autonomous ordinary differential equations in the unknowns $u_{i}$ and $v_{i}$.

For small value of $\varepsilon$, Eqs. (44) and (45) can be averaged introducing a limited error. Integrating each function with respect to $t$ from 0 to $T=2 \pi / \omega$ while holding $u_{i}$ and $v_{i}$ constant, we obtain the autonomous system ${ }^{5}$

$$
\begin{aligned}
\dot{u}_{i}= & -\frac{\varepsilon}{2 \omega}\left(\gamma_{i} \omega u_{i}+\frac{3}{4} \delta_{i}\left(u_{i}^{2}+v_{i}^{2}\right) v_{i}+\Omega_{i} v_{i}+\sigma_{i} \omega u_{j}\right) \\
\dot{v}_{i}= & -\frac{\varepsilon}{2 \omega}\left(A_{i}+\gamma_{i} \omega v_{i}-\frac{3}{4} \delta_{i}\left(u_{i}^{2}+v_{i}^{2}\right) u_{i}-\Omega_{i} u_{i}\right. \\
& \left.+\sigma_{i} \omega v_{j}\right)
\end{aligned}
$$

Introducing polar coordinates

$u_{i}=a_{i} \cos \varphi_{i} \quad v_{i}=a_{i} \sin \varphi_{i}$,

a straightforward calculation gives

$$
\begin{aligned}
\dot{a}_{i}= & -\frac{\varepsilon}{2 \omega}\left(A_{i} \sin \varphi_{i}+\gamma_{i} \omega a_{i}+\sigma_{i} \omega a_{j} \cos \left(\varphi_{i}-\varphi_{j}\right)\right) \\
\dot{\varphi}_{i}= & -\frac{\varepsilon}{2 \omega}\left(\Omega_{i}-\frac{A_{i}}{a_{i}} \cos \varphi_{i}+\frac{3}{4} \delta_{i} a_{i}^{2}\right. \\
& \left.+\sigma_{i} \omega \frac{a_{j}}{a_{i}} \sin \left(\varphi_{i}-\varphi_{j}\right)\right)
\end{aligned}
$$

The equilibrium points of equations (49) and (50) correspond to oscillations of constant amplitude in the original equations. They are found solving the system $\left(\psi=\varphi_{2}-\varphi_{1}\right)$

$$
A \sin \varphi_{1}+\gamma_{1} \omega a_{1}+\sigma_{1} \omega a_{2} \cos \psi=0
$$

$\gamma_{2} \omega a_{2}-\sigma_{2} \omega a_{1} \cos \psi=0$

$$
-A \cos \varphi_{1}+\frac{3}{4} \delta_{1} a_{1}^{3}+\Omega_{1} a_{1}-\sigma_{1} \omega a_{2} \sin \psi=0
$$

$\frac{3}{4} \delta_{2} a_{2}^{3}+\Omega_{2} a_{2}-\sigma_{2} \omega a_{1} \sin \psi=0$

Equations (52) and (54) imply

$$
\cos \psi=\frac{\gamma_{2}}{\sigma_{2}} \frac{a_{2}}{a_{1}}
$$

\footnotetext{
5 With some abuse of notation, we use the same symbols $u_{i}$ and $v_{i}$ to denote also the averaged quantities.
}

$\sin \psi=\frac{\frac{3}{4} \delta_{2} a_{2}^{3}+\Omega_{2} a_{2}}{\sigma_{2} \omega a_{1}}$

Squaring and summing, we obtain the first equation for the steady-state amplitudes

$\gamma_{2}^{2} \omega^{2} a_{2}^{2}+\left(\frac{3}{4} \delta_{2} a_{2}^{3}+\Omega_{2} a_{2}\right)^{2}-\sigma_{2}^{2} \omega^{2} a_{1}^{2}=0$

Substituting (55) and (56) into (51) and (53), respectively, rearranging the terms, squaring and summing, we find the second amplitude equation

$$
\begin{aligned}
- & A^{2} a_{1}^{2}+\left(\gamma_{1} \omega a_{1}^{2}+\gamma_{2} \omega \frac{\sigma_{1}}{\sigma_{2}} a_{2}^{2}\right)^{2}+ \\
& +\left(\frac{3}{4} \delta_{1} a_{1}^{4}+\Omega_{1} a_{1}^{2}-\frac{\sigma_{1}}{\sigma_{2}} a_{2}\left(\frac{3}{4} \delta_{2} a_{2}^{3}+\Omega_{2} a_{2}\right)\right)^{2}=0
\end{aligned}
$$

The nonlinear algebraic system (57)-(58) can be solved numerically, finding the steady-state amplitudes $a_{1}$ and $a_{2}$. These, in turn, allow to compute the phase shifts $\varphi_{1}$ and $\varphi_{2}$ through (55)-(56), and finally the state variables

$$
\begin{aligned}
& i_{1}(t)=\dot{x}_{1}(t)=-\omega a_{1} \sin \left(\omega t+\varphi_{1}\right) \\
& \left.\begin{array}{l}
v_{2}(t) \\
i_{2}(t)
\end{array}\right\}=\dot{x}_{2}(t)=-\omega a_{2} \sin \left(\omega t+\varphi_{2}\right)\left\{\begin{array}{l}
\text { for } \mathrm{pz} \\
\text { for } \mathrm{em}
\end{array}\right.
\end{aligned}
$$

The average injected power for both the piezoelectric and the electromagnetic energy harvester is

$$
\begin{aligned}
P_{\text {in }} & =\frac{1}{T} \int_{0}^{T} v_{\text {in }}(t) \dot{q}_{1}(t) \mathrm{d} t \\
& =-\frac{\varepsilon A L_{1} \omega a_{1}}{T} \int_{0}^{T} \sin \left(\omega t+\varphi_{1}\right) \cos (\omega t) \mathrm{d} t \\
& =-\frac{\varepsilon A L_{1} \omega a_{1}}{2} \sin \varphi_{1}=\frac{\varepsilon L_{1} \omega^{2}}{2 \sigma_{2}}\left(\gamma_{1} \sigma_{2} a_{1}^{2}+\gamma_{2} \sigma_{1} a_{2}^{2}\right)
\end{aligned}
$$

where the inverse of (41) has been used, together with (51) and (55).

The average dissipated power for the piezoelectric harvester is

$$
\begin{aligned}
P_{\mathrm{out}}^{\mathrm{pz}} & =\frac{1}{T} \int_{0}^{T} \frac{v_{2}(t)^{2}}{R_{2}} \mathrm{~d} t \\
& =\frac{\omega^{2} a_{2}^{2}}{T R_{2}} \int_{0}^{T} \sin ^{2}\left(\omega t+\varphi_{2}\right) \mathrm{d} t=\frac{\omega^{2} a_{2}^{2}}{2 R_{2}}
\end{aligned}
$$




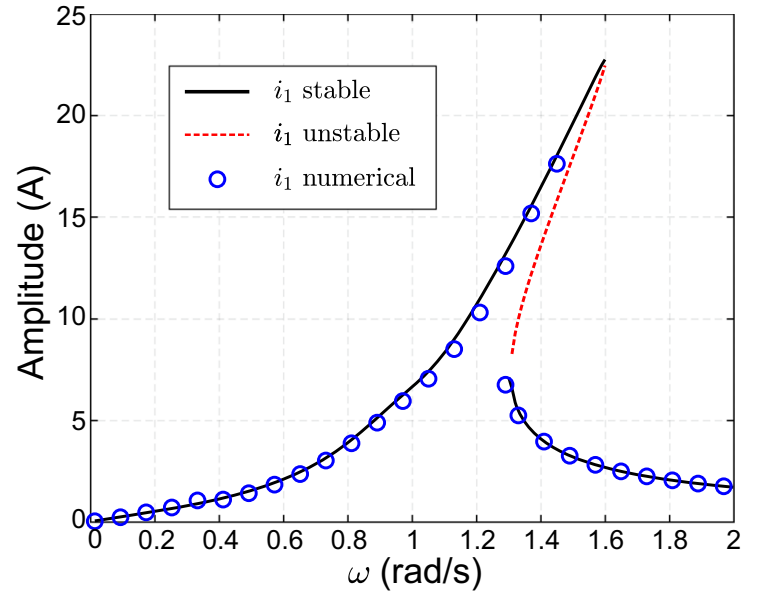

Fig. 10 Input current amplitude for the nonlinear coupled resonators. Lines derive from the theoretical analysis, while symbols result from numerical simulations: Blue circles refer to the resistive-inductive load. Parameters are $\delta_{1}=0.1, \delta_{2}=0.5$, $V_{\text {in }}=1 \mathrm{~V}$, and other parameters are the same as in Fig. 5 . (Color figure online)

with power efficiency

$\eta_{\mathrm{pz}}=\frac{\sigma_{2} G_{2} a_{2}^{2}}{\varepsilon L_{1}\left(\gamma_{1} \sigma_{2} a_{1}^{2}+\gamma_{2} \sigma_{1} a_{2}^{2}\right)}$

For the electromagnetic energy harvester, the average dissipated power is

$P_{\mathrm{out}}^{\mathrm{em}}=\frac{1}{T} \int_{0}^{T} R_{2} \dot{q}_{2}(t)^{2} \mathrm{~d} t=\frac{\omega^{2} R_{2} a_{2}^{2}}{2}$

with power efficiency

$\eta_{\mathrm{em}}=\frac{\sigma_{2} R_{2} a_{2}^{2}}{\varepsilon L_{1}\left(\gamma_{1} \sigma_{2} a_{1}^{2}+\gamma_{2} \sigma_{1} a_{2}^{2}\right)}$

To confirm our theoretical prediction, we have performed extensive numerical simulations. The following results refer to the case of the piezoelectric EH model. Based on the analogy shown at the beginning of this section, the results can be easily adapted to the case of electromagnetic energy harvester.

Figures 10 and 11 show the amplitude response for the current $i_{1}(t)$ and the voltage $v_{2}(t)$, respectively, as a function of the voltage source angular frequency $\omega$, for the piezoelectric nonlinear energy harvester.

As expected, both curves show the hysteretic behavior due to the nonlinear stiffness. Stability and bifurca-

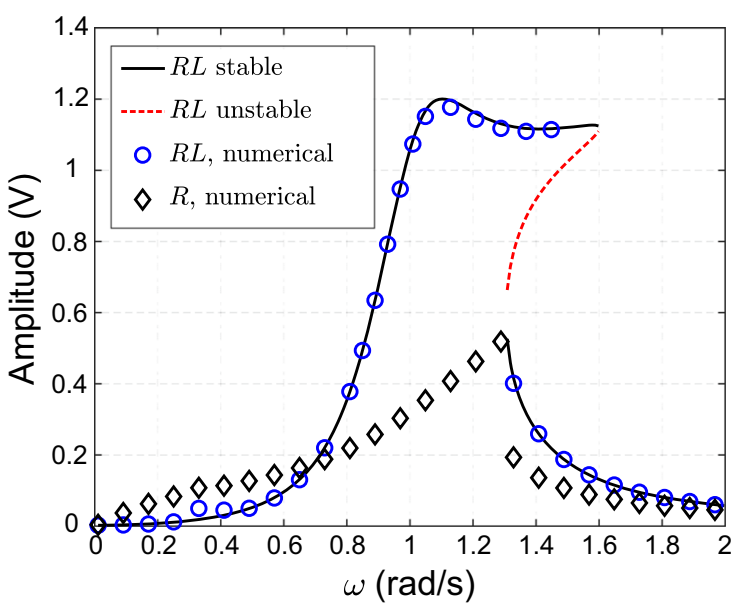

Fig. 11 Output voltage amplitude comparison for a nonlinear resonator-based energy harvester: Resistive load versus resistive-inductive load. Black diamonds refer to the resistive load. Parameters are the same as in Fig. 10

tion analysis for the averaged system are determined computing the eigenvalues of the Jacobian matrix of system (49)-(50). For small values of the forcing frequency, there is only one solution, which is a stable equilibrium point of the averaged system and corresponds to stable periodic oscillations of $i_{1}(t)$ and $v_{2}(t)$ (increasing full black line), with the same period of the forcing term. At the critical value $\omega_{S N_{1}} \simeq 1.31$, a saddle node bifurcation, identified by a null eigenvalue, occurs. Two new, initially coincident, equilibrium points emerge that separate and become distinct when $\omega$ is further increased. The smaller amplitude corresponds to a stable equilibrium point (decreasing full line), while the larger amplitude is a saddle (dashed line). At the critical value $\omega_{S N_{2}} \simeq 1.57 \mathrm{rad} / \mathrm{s}$, the unstable saddle collides with the larger, stable equilibrium point, and they vanish through a second saddle node bifurcation. The small, stable equilibrium point ultimately survives as the only solution. Theoretical predictions of our perturbation approach are compared with the results obtained from the numerical integration of equations (32)-(33) (symbols). Numerical solutions were obtained applying a continuation method, using the solution for a certain value of the forcing frequency as the initial condition for the following value of this parameter. Obviously, the saddle-type limit cycle cannot be detected neither through forward nor backward time integration. Moreover, as the parameter $\omega$ approaches the critical value $\omega_{S N_{2}}$, it becomes 
increasingly difficult to follow the large stable solution, because its basin of attraction becomes smaller (this solution and the unstable saddle-type limit cycle get closer to each other), and trajectories are attracted toward the small stable limit cycle.

For comparison purposes, Fig. 11 also shows the output voltage for the resistive load case. This value has been calculated solving numerically the ODE system (2)-(3) for the resistive load. The output voltage curve shows a clear resemblance to the linear case, though with a high, long right tale. This behavior is due to the resonance between the output voltage $v_{2}(t)$ and the nonlinear hysteretic behavior of the current $i_{1}(t)$. The maximum amplitude is also shifted toward higher frequency values. More importantly, the matched $R L$ load case clearly outperforms the resistive load case in a wide frequency interval.

Figure 12 shows the output average power as a function of the forcing frequency, for both the resistivereactive and the purely resistive load. For the $R L$ load, we have calculated the output power for both the stable (full line) and the unstable (dashed line) limit cycles, although only stable solutions are accessible for the system. The theoretical result given by (62) is compared with the numerical values obtained evaluating

$P_{\text {out }}=\frac{1}{T} \int_{0}^{T} G_{2} \dot{\phi}_{2}^{2}(t) \mathrm{d} t$

where $T=2 \pi / \omega$ is the period of the oscillation, and $\dot{\phi}_{2}(t)$ is calculated through numerical integration of the state equations (32)-(33) (symbols). It is worth noticing that the nonlinearity introduces a double peak in the average output power.

In the nonlinear case, the optimal value of the load resistance $R_{2}$ maximizing the average dissipated power cannot be found analytically. In fact, $R_{2}$ influences the value of $a_{2}$, which is found numerically and that, in turns, determines the output power. The dependence of the average output power and power efficiency on the output conductance is shown in Figs. 13 and 14, respectively, for a fixed value of the input frequency $\omega$. Both the cases of resistive-reactive and purely resistive load are considered. Again, the $R L$ load solution determines a larger average output power together with a better efficiency over the whole range of values considered for $G_{2}$. Notice also that maximum power and power efficiency are obtained at a lower conductance with respect to the linear case.

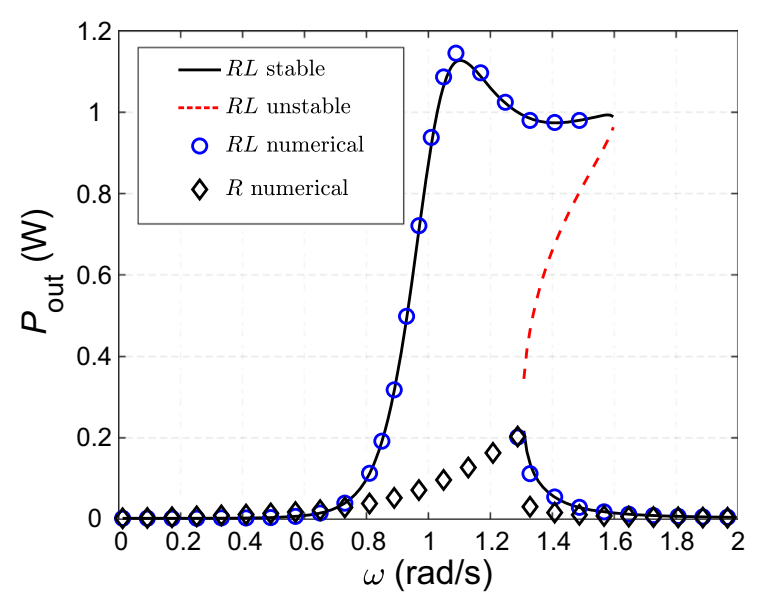

Fig. 12 Output average power as a function of forcing frequency, for both the resistive-reactive $R L$ load, and the resistive $R$ load. Parameters are the same as in Fig. 10

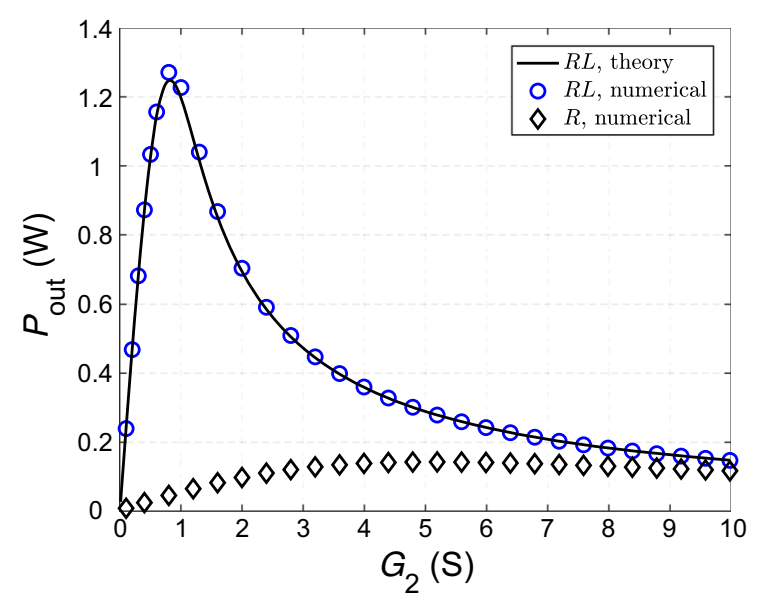

Fig. 13 Output average power as a function of the load conductance $G_{2}$ for $\omega=1$. Solid line (theoretical result from (62)) and circles (numerical simulations) refer to the resistive-inductive load, and red diamonds refer to the resistive load (numerical simulations only). Parameters are the same as in Fig. 10

In Fig. 15, we show the power efficiency as a function of the source angular frequency $\omega$, here represented for the optimal value of $G_{2}$ valid for the linear harvester, i.e., $G_{2}=k_{v} k_{c} / R_{1}=1.56 \mathrm{~S}$. Even for the nonlinear case, the efficiency reaches an upper limit of about $50 \%$ at the resonance frequency, showing a very good agreement between the theoretical prediction from (63) and the results of the numerical simulations. It is worth noticing that although the average output power shows a double peak in Fig. 12, the efficiency is single peaked, 


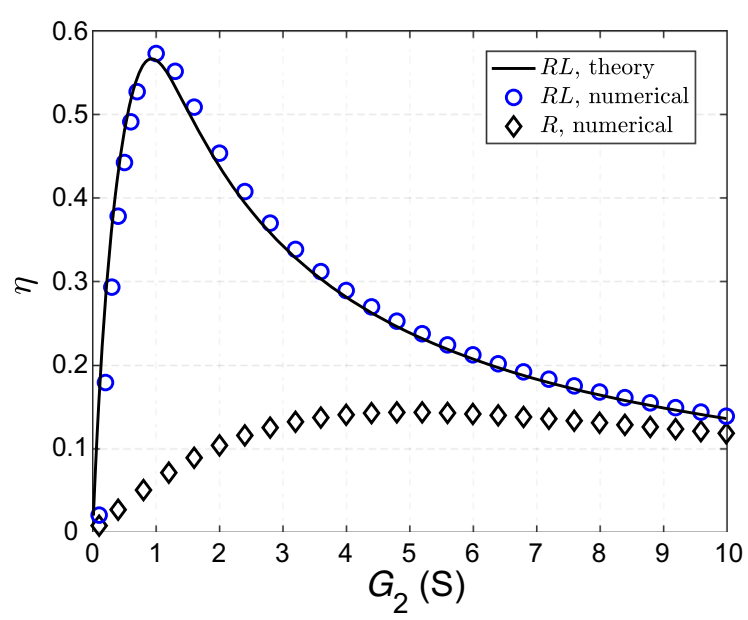

Fig. 14 Power efficiency comparison as a function of the load conductance $G_{2}$ for $\omega=1$. Solid line (theoretical result from (62)) and circles (numerical simulations) refer to the resistiveinductive load, and black diamonds refer to the resistive load (numerical simulations only). Parameters are the same as in Fig. 10

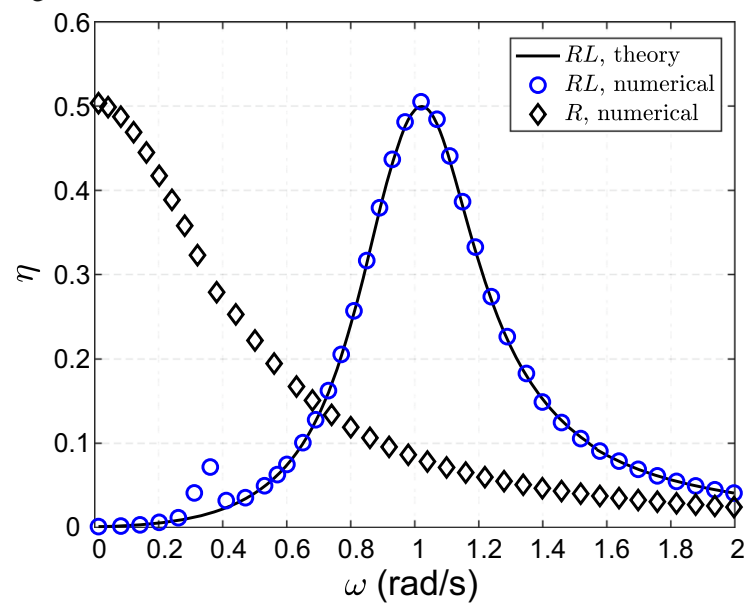

Fig. 15 Power efficiency comparison as a function of the forcing frequency. Solid line (theoretical result from (63)) and circles (numerical simulations) refer to the resistive-inductive load, and red diamonds refer to the resistive load (numerical simulations only). Parameters are the same as in Fig. 10

because the average input power is strictly increasing up to the bifurcation frequency $\omega_{S N_{2}}$.

For comparison, we also show the result of the numerical simulations for the purely resistive load, which is almost identical to the linear case, being the system only weakly nonlinear.

Finally, Fig. 16 shows the average power delivered to the load (above), and the power efficiency (below), as functions of the load inductance $L_{2}$, for the nonlin-
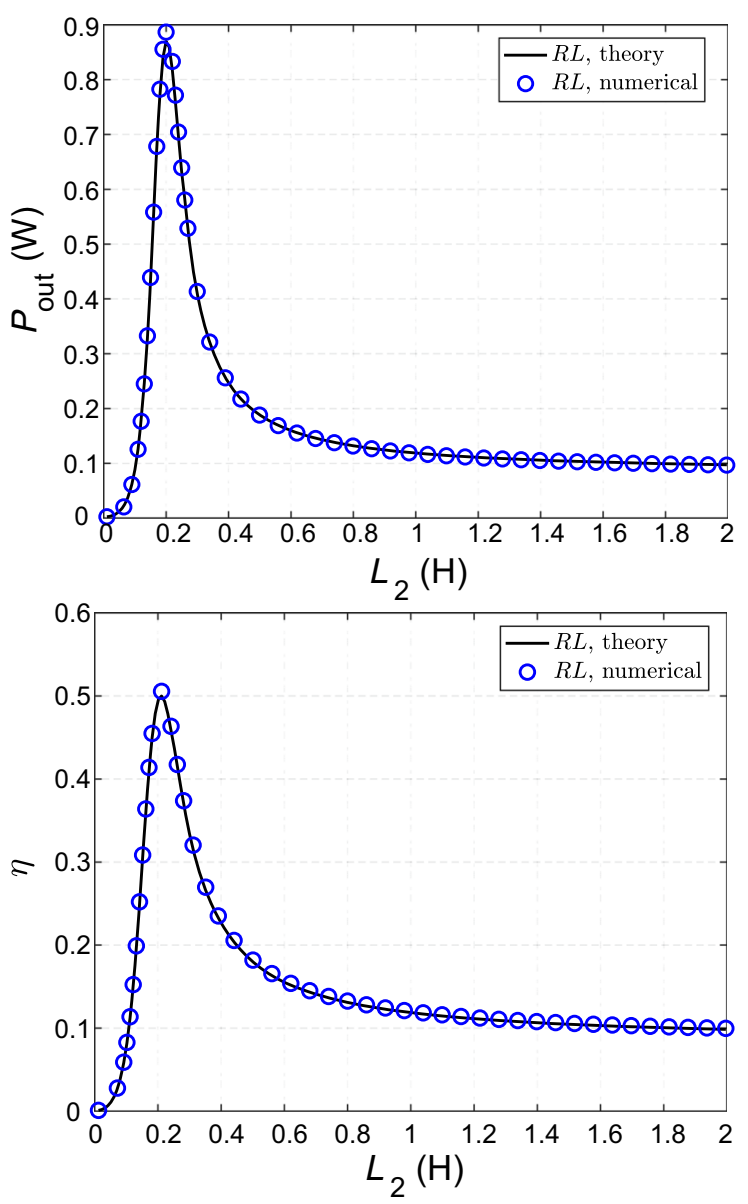

Fig. 16 Average power delivered to the load (above) and power efficiency (below) as functions of the load inductance $L_{2}$, for $\omega=1$. Solid lines are theoretical result from (62) and (63), respectively. Blue circles refer to numerical simulations. Parameters are the same as in Fig. 10

ear piezoelectric harvester. Theoretical predictions are obtained solving (57) and (58), and then, $P_{\text {out }}$ and $\eta$ are computed from (62) and (63), respectively. Results show a good agreement with the numerical experiments. Because the system is weakly nonlinear, the curves show a clear resemblance with the corresponding curves for the linear harvester. It can be seen that both $P_{\text {out }}$ and $\eta$ reach a maximum for $L_{2}$ close to the optimal value for linear systems $L_{2}=L 1 C_{1} / C_{2}$. Moreover, both the average power and efficiency converge to constant values for large values of $L_{2}$. As explained for the linear system, this is due to the fact that the inductor becomes equivalent to an open circuit for $L_{2} \rightarrow+\infty$. 


\section{Conclusions}

We have analyzed vibrational energy harvesting systems with piezoelectric and magnetic inductive transducers, assuming the power of external disturbance concentrated around a specific frequency.

Typically, energy harvesters are modeled as oscillators, either linear or nonlinear, coupled to first-order systems that describe the transducer's dynamics. For linear harvester, we used circuit theory to show that the harvested energy can be maximized by a proper matching of the load, usually assumed to be a simple resistor. Analogously to the power factor correction procedure, a reactive passive component can be added that maximizes the average power delivered to the load. As a result, the energy harvester is modeled by two coupled oscillators that must be designed to have the same resonance frequency. This solution allows for maximum power collection by the non-degenerate resistive load in the presence of finite losses of the harvester. Moreover, the maximum $50 \%$ efficiency under maximum power transfer condition can be achieved at the same resonant frequency.

For the nonlinear system, we used nonlinear dynamics methods to derive analytical formulae for the output, the harvested energy and the power efficiency. In full analogy with the linear case, modifying the load increases the harvested energy and power efficiency. As opposed to the linear case, however, the efficiency can reach values above $50 \%$, again assuming realistic values for both the load and the harvester losses.

Funding Open access funding provided by Politecnico di Torino within the CRUI-CARE Agreement.

\section{Compliance with ethical standard}

Conflict of interest The authors declare that they have no conflict of interest.

Open Access This article is licensed under a Creative Commons Attribution 4.0 International License, which permits use, sharing, adaptation, distribution and reproduction in any medium or format, as long as you give appropriate credit to the original author(s) and the source, provide a link to the Creative Commons licence, and indicate if changes were made. The images or other third party material in this article are included in the article's Creative Commons licence, unless indicated otherwise in a credit line to the material. If material is not included in the article's Creative Commons licence and your intended use is not permitted by statutory regulation or exceeds the permitted use, you will need to obtain permission directly from the copyright holder. To view a copy of this licence, visit http://creativecommons.org/licenses/by/4.0/.

\section{Appendix: State equations for the electrical domain}

In this "Appendix," we derive the state equations for both the mechanical and electrical domains of the piezoelectric and the electromagnetic energy harvesters.

For the piezoelectric energy harvester, the state equations for the electromechanical system are (1), (3) that we rewrite in the form

$$
\begin{aligned}
\ddot{x} & =-\frac{U^{\prime}(x)}{m}-\frac{\gamma}{m} \dot{x}-\frac{k_{v}}{m} v_{2}+\frac{1}{m} F_{\text {in }} \\
\dot{v}_{2} & =\frac{k_{c}}{C_{2}} \dot{x}-\frac{1}{C_{2}} i_{2}
\end{aligned}
$$

For the resistive load in Fig. 8 a, $i_{2}=G_{2} v_{2}$, thus obtaining the electromechanical model equations in [18,33]. For the $R L$ load in Fig. $8 \mathbf{b}$, applying KCL yields $i_{2}=G_{2} v+i_{L_{2}}$. Using the constitutive relation for the inductor $\phi_{2}=L_{2} i_{L_{2}}$, and the flux-voltage relationship $\dot{\phi}_{2}=v$, equations (67) become

$$
\begin{aligned}
\ddot{x} & =-\frac{U^{\prime}(x)}{m}-\frac{\gamma}{m} \dot{x}-\frac{k_{v}}{m} \dot{\phi}_{2}+\frac{1}{m} F_{\text {in }} \\
\ddot{\phi}_{2} & =\frac{k_{c}}{C_{2}} \dot{x}-\frac{1}{L_{2} C_{2}} \phi_{2}-\frac{G_{2}}{C_{2}} \dot{\phi}_{2}
\end{aligned}
$$

For the electromagnetic energy harvester, the electromechanical equations are (4) and (6) that we rewrite in the form

$$
\begin{aligned}
\ddot{y} & =-\frac{U^{\prime}(y)}{m}-\frac{c}{m} \dot{y}-\frac{r_{1}}{m} i_{2}+\frac{F_{\text {in }}}{m} \\
\dot{i}_{2} & =\frac{r_{2}}{L_{2}} \dot{y}-\frac{v_{2}}{L_{2}}
\end{aligned}
$$

For the resistive load in Fig. $8 \mathbf{a}, v_{2}=R_{2} i_{2}$.

For the $R C$ load in Fig. 8 c, applying KVL yields $v_{2}=R_{2} i_{2}+v_{C_{2}}$. Using the constitutive relation for the capacitor $q_{2}=C_{2} v_{C_{2}}$, and the current-charge relationship $\dot{q}_{2}=i_{2}$, equation (69) becomes

$$
\begin{aligned}
\ddot{y} & =-\frac{U^{\prime}(y)}{m}-\frac{c}{m} \dot{y}-\frac{r_{1}}{m} \dot{q}_{2}+\frac{F_{i n}}{m} \\
\ddot{q}_{2} & =\frac{r_{2}}{L_{2}} \dot{y}-\frac{1}{L_{2} C_{2}} q_{2}-\frac{R_{2}}{L_{2}} \dot{q}_{2}
\end{aligned}
$$




\section{References}

1. Internet of things (IoT) connected devices installed base worldwide from 2015 to 2025 . https://www.statista.com/ statistics/471264/iot-number-of-connected-devices-world wide/. Accessed: 2020, July 19

2. Ashton, K., et al.: That 'internet of things' thing. RFID J. 22(7), 97-114 (2009)

3. Atzori, L., Iera, A., Morabito, G.: The internet of things: A survey. Comput. Netw. 54(15), 2787-2805 (2010)

4. Tan, L., Wang, N.: Future internet: the internet of things. In: 2010 3rd International Conference on Advanced Computer Theory and Engineering (ICACTE), volume 5, pp. V5-376. IEEE (2010)

5. Paradiso, J.A., Starner, T.: Energy scavenging for mobile and wireless electronics. IEEE Pervasive Comput. 4(1), 18-27 (2005)

6. Yang, G.-Z. (ed.): Body Sensor Networks, vol. 1. Springer, Berlin (2006)

7. Kurs, A., Karalis, A., Moffatt, R., Joannopoulos, J.D., Fisher, P., Soljačić, M.: Wireless power transfer via strongly coupled magnetic resonances. Science 317(5834), 83-86 (2007)

8. Cannon, B.L., Hoburg, J.F., Stancil, D.D., Goldstein, S.C.: Magnetic resonant coupling as a potential means for wireless power transfer to multiple small receivers. IEEE Trans. Power Electron. 24(7), 1819-1825 (2009)

9. Sample, A.P., Meyer, D.T., Smith, J.R.: Analysis, experimental results, and range adaptation of magnetically coupled resonators for wireless power transfer. IEEE Trans. Industr. Electron. 58(2), 544-554 (2010)

10. Roundy, S., Wright, P.K., Rabaey, J.M.: Energy Scavenging for Wireless Sensor Networks. Springer, Berlin (2003)

11. Priya, S., Inman, D.J.: Energy Harvesting Technologies, vol. 21. Springer, Berlin (2009)

12. Erturk, A., Inman, D.J.: Piezoelectric Energy Harvesting. Wiley, Hoboken (2011)

13. Khaligh, A., Zeng, P., Zheng, C.: Kinetic energy harvesting using piezoelectric and electromagnetic technologies-state of the art. IEEE Trans. Industr. Electron. 57(3), 850-860 (2009)

14. Vocca, H., Neri, I., Travasso, F., Gammaitoni, L.: Kinetic energy harvesting with bistable oscillators. Appl. Energy 97, 771-776 (2012)

15. Wen, X., Yang, W., Jing, Q., Wang, Z.L.: Harvesting broadband kinetic impact energy from mechanical triggering/vibration and water waves. ACS Nano 8(7), 7405-7412 (2014)

16. Fu, Y., Ouyang, H., Davis, R.B.: Nonlinear dynamics and triboelectric energy harvesting from a three-degree-offreedom vibro-impact oscillator. Nonlinear Dyn. 92(4), 1985-2004 (2018)

17. Cottone, F., Vocca, H., Gammaitoni, L.: Nonlinear energy harvesting. Phys. Rev. Lett. 102(8), 080601 (2009)

18. Gammaitoni, L., Neri, I., Vocca, H.: Nonlinear oscillators for vibration energy harvesting. Appl. Phys. Lett. 94(16), 164102 (2009)

19. Mann, B.P., Sims, N.D.: Energy harvesting from the nonlinear oscillations of magnetic levitation. J Sound Vib. 319(1-2), 515-530 (2009)
20. Gammaitoni, L., Neri, I., Vocca, H.: The benefits of noise and nonlinearity: extracting energy from random vibrations. Chem. Phys. 375(2), 435-438 (2010)

21. Daqaq, M.F.: Response of uni-modal duffing-type harvesters to random forced excitations. J. Sound Vib. 329(18), 3621-3631 (2010)

22. Bonnin, M., Traversa, F.L., Bonani, F.: Analysis of influence of nonlinearities and noise correlation time in a single-DOF energy-harvesting system via power balance description. Nonlinear Dyn. 100(1), 119-133 (2020)

23. Daqaq, M.F.: On intentional introduction of stiffness nonlinearities for energy harvesting under white Gaussian excitations. Nonlinear Dyn. 69(3), 1063-1079 (2012)

24. Ming, X., Li, X.: Two-step approximation procedure for random analyses of tristable vibration energy harvesting systems. Nonlinear Dyn. 98(3), 2053-2066 (2019)

25. Daqaq, M.F., Crespo, R.S., Ha, S.: On the efficacy of charging a battery using a chaotic energy harvester. Nonlinear Dyn. 99(2), 1525-1537 (2020)

26. Yan, Z., Sun, W., Hajj, M.R., Zhang, W., Tan, T.: Ultrabroadband piezoelectric energy harvesting via bistable multi-hardening and multi-softening. Nonlinear Dyn. 100, 1057-1077 (2020)

27. Renno, J.M., Daqaq, M.F., Inman, D.J.: On the optimal energy harvesting from a vibration source. J. Sound Vib. 320(1-2), 386-405 (2009)

28. Abdelmoula, H., Abdelkefi, A.: Ultra-wide bandwidth improvement of piezoelectric energy harvesters through electrical inductance coupling. Eur. Phys. J. Spec. Top. 224(14-15), 2733-2753 (2015)

29. Yan, B., Zhou, S., Litak, G.: Nonlinear analysis of the tristable energy harvester with a resonant circuit for performance enhancement. Int. J. Bifurc. Chaos 28(07), 1850092 (2018)

30. Huang, D., Zhou, S., Litak, G.: Analytical analysis of the vibrational tristable energy harvester with a RL resonant circuit. Nonlinear Dyn. 97(1), 663-677 (2019)

31. Zhou, S., Tianjun, Yu.: Performance comparisons of piezoelectric energy harvesters under different stochastic noises. AIP Adv. 10(3), 035033 (2020)

32. Masana, D.J., Daqaq, M.F.: Electromechanical modeling and nonlinear analysis of axially loaded energy harvesters. J. Vib. Acoust. 133(1), (2011)

33. Lefeuvre, E., Badel, A., Richard, C., Petit, L., Guyomar, D.: A comparison between several vibration-powered piezoelectric generators for standalone systems. Sens. Actuators A 126(2), 405-416 (2006)

34. Loo, C., Secord, N.: Computer models for fading channels with applications to digital transmission. IEEE Trans. Veh. Technol. 40(4), 700-707 (1991)

35. Kwon, S.-D., Park, J., Law, K.: Electromagnetic energy harvester with repulsively stacked multilayer magnets for low frequency vibrations. Smart Mater. Struct. 22(5), 055007 (2013)

36. Kucab, K., Górski, G., Mizia, J.: Energy harvesting in the nonlinear electromagnetic system. Eur. Phys. J. Spec. Top. 224(14-15), 2909-2918 (2015)

37. Gammaitoni, L.: There's plenty of energy at the bottom (micro and nano scale nonlinear noise harvesting). Contemp. Phys. 53(2), 119-135 (2012) 
38. Chua, L.O., Desoer, C.A., Kuh, E.S.: Linear and Nonlinear Circuits. McGraw-Hill, New York (1987)
39. Yang, Z., Erturk, A., Jean, Z.: On the efficiency of piezoelectric energy harvesters. Extreme Mech. Lett. 15, 26-37 (2017)

40. Guckenheimer, J., Holmes, P.: Nonlinear Oscillations, Dynamical Systems, and Bifurcations of Vector Fields, vol. 42. Springer, Berlin (2013)

Publisher's Note Springer Nature remains neutral with regard to jurisdictional claims in published maps and institutional affiliations. 